citation, the cross section $\sigma_{\mathrm{v}}(E)$ is represented by the line spectrum of Fig. 4 of this paper, which has a centre of intensity at $E_{0}=-6 \mathrm{meV}$ in relative resonance energies as already mentioned in the text. One gets the maximum of inelastically scattered electrons for $E_{\mathrm{g}}=E_{0}$ and the maximum of detected current, if also $E_{\mathrm{a}}=E_{0}-W$, where $W$ is the energy loss of the scattered electrons. The elastic cross section $\sigma_{\mathrm{el}}(E)$ can be assumed as nearly constant over the energy range of the functions $f_{\mathrm{g}}$ and $f_{\mathrm{a}}$. Under these conditions and with the absolute elastic cross section of Fig. 6, one gets from the ratio of the peak maxima in Fig. 1: $S_{v=1}^{\prime}=6.7 \cdot 10^{-20} \mathrm{~cm}^{2} \mathrm{eV} /$ ster. We have assumed Gaussian profiles for $f_{\mathrm{g}}$ and $f_{\mathrm{a}}$, the half widths of which were $\Delta E_{\mathrm{g}}=58 \mathrm{meV}$ and
$\Delta E_{\mathrm{a}}=31 \mathrm{meV}$, respectively, in accordance with the data of the selectors (slit width, mean radius, transmision energy). The measured half widths of the elastic peak $(\triangle E=65 \mathrm{meV})$ and the inelastic peak $(\Delta E=35 \mathrm{meV}$ ) in Fig. 1 are consistent with all assumptions of this appendix.

In $S_{v=1}^{\prime}$, the outer lines of the structure of Fig. 4 are suppressed by the energy profiles $f_{\mathrm{g}}$ and $f_{\mathrm{a}}$. A correction of $20 \%$ must be applied to get the energy-integrated cross section

$$
S_{v=1}=\int \sigma_{v=1}(E) \mathrm{d} E,
$$

which is the desired final result. This gives $S_{v=1}=$ $8 \cdot 10^{-20} \mathrm{~cm}^{2} \mathrm{eV} / \mathrm{ster}$ which corresponds to the area under the resonance structure of Figure 4.

\title{
Interaction of Randomly Phased Vibrational Waves via the Collision Dominated Solid State Plasma
}

\author{
W. WONNEBERGER \\ Physikalisches Institut der Universität Freiburg i. Br. \\ (Z. Naturforsch. 26 a, 1625-1629 [1971] ; received 24, July 1971)
}

\begin{abstract}
A diagrammatic technic is presented which is appropriate to study the nonlinear response of the collision dominated solid state plasma to randomly phased ac fields. By summation of diagrams, a nonlinear integral equation is established for the propagator $S^{\prime}$ which is directly related to the nonlinear response function. Using the equation for $S^{\prime}$, an equivalence is derived between a model solution in the theory of nonlinear coherent ultrasound amplification and the interaction theory of a great number of randomly phased vibrational modes, confined to a small frequency band.
\end{abstract}

\section{Introduction}

It has been proposed by Hutson ${ }^{1}$ that the interaction of highly amplified vibrational waves in a piezoelectric semiconductor predominantly proceeds via the electron gas by means of the concentration nonlinearity. An ultrasonic wave produces an accompanying electric field which perturbs the electron gas. The electron gas usually is in the state of a collision dominated plasma. In this case, the Coulomb interaction of the electrons manifests itself in form of the concentration nonlinearity. The theory of vibrational wave interactions in a piezoelectric semiconductor thus essentially is equivalent to the study of the nonlinear response of the collision dominated solid state plasma.

1 A. R. Hutson, Phys. Rev. Lett. 9, 296 [1962].

2 K. Yamada, Phys. Rev. 169, 690 [1968].

3 V. L. Gurevich, V. D. Kagan, and B. D. Laikhtman, Sov. Phys. JETP 27, 102 [1968].

4 B. K. Ridley and J. Wilkinson, Brit. J. Phys. C 2, 1307 [1969].
In recent years, the following special case has attracted some attention: The great number of acoustoelectrically active modes present in the crystal may be treated in the random phase approximation (RPA). More precisely, one assumes that these waves are subject to a Gaussian random process. One of the first investigators to treat this problem, were YAmada ${ }^{2}$ and Gurevich et al. ${ }^{3}$ who derived on this basis transpont equations for the phonon distribution function. Recently, RIDLEY and WILKINSON ${ }^{4}$, GANGUly and Conwell ${ }^{5}$, and Butcher and SleCHTA ${ }^{6}$ investigated the acoustoelectric gain of vibrational waves within the RPA.

All these authors confine themselves to the lowest orders in perturbation theory.

5 A. K. Ganguly and E. M. Conwell, Phys. Lett. 29 A, 271 [1969].

6 P. N. Butcher and J. Slechta, Brit. J. Phys. C 4, 870 [1971]. 
In the present paper, we devise a diagrammatic method capable of treating this problem to an arbitrarily high order in perturbation theory. This method is used to derive an integral equation for the renormalized propagator which is associated with the nonlinear response function of the collision dominated solid state plasma. Using this result, we are able, to derive an equivalence between a model solution in the theory of nonlinear coherent sound amplification and a simple multimode situation, namely when a very great number of randomly phased modes are concentrated in a small frequency band. We thus substantiate an early statement in Ref. ${ }^{7}$.

\section{Basic Equations}

The object of our interest is the collision dominated solid state plasma. We therefore start from the nonlinear current density equation, which in quasi static approximation and in one dimension may be written (cf. Ref. ${ }^{7}$ ) :

$$
\begin{aligned}
& \tilde{\varrho}(v Q)=S(v) \sum_{x} x \frac{\varphi(x Q)}{k_{\mathrm{B}} T} \tilde{\varrho}((v-\varkappa) Q) \quad v \neq 0, \\
& \tilde{\varrho}(0)=n_{0} .
\end{aligned}
$$

Here, $\tilde{\varrho}(v Q)$ is the electronic density fluctuation associated with the wave vector $(v Q)$ and $\tilde{\varphi}$ is the corresponding piezoelectric potential. The tilde indicates that frequency factors $\exp \{-i \omega(\nu Q) t\}$ have been omitted. $S(v)$ is given by

$$
S(v)=\left[v-i \frac{\omega_{\mathrm{D}}}{\omega(Q)}\left(1-\frac{v_{\mathrm{d}}}{v_{\mathrm{s}}}\right)\right]^{-1},
$$

where $\omega_{\mathrm{D}}$ is the diffusion frequency, $v_{\mathrm{s}}$ the sound velocity and $v_{\mathrm{d}}$ the electronic drift velocity. $n_{0}$ is the dopant concentration. Following the method of Butcher and Slechta ${ }^{6} Q$ is itaken to be a very small quantity such that an arbitrarily wave vector $k$ may be represented as $k=\varkappa(k) Q$ where $\varkappa(k)$ is an appropriate integer which eventually is very high. Thus $Q$ may be chosen as the wave number resolution in an experiment on the spectral distribution of acoustoelectrically amplified noise.

Equation (1) may be cast into still a simpler form. Extracting the $x=\boldsymbol{v}$ term from the sum (in which from now on terms with vanishing wave vectors $\varkappa=0$ and $\varkappa^{\prime}=0$ are excluded from the sum

\footnotetext{
7 W. Wonneberger, Phys. Stat. Sol. 40, 127 [1970].
}

which is indicated by $\Sigma^{\prime}$ ) one obtains after substituting

$$
\frac{\tilde{\varrho}(v Q)}{n_{0}}=r(v), \quad \varkappa \frac{\hat{\varphi}(\varkappa Q)}{k_{\mathrm{B}} T}=f(\varkappa)
$$

the relation

$$
\begin{array}{ll}
r(v)=S(v) f(v)+S(v) \sum_{\varkappa+\varkappa^{\prime}=v}^{\prime} f(\varkappa) r\left(\varkappa^{\prime}\right) & v \neq 0, \\
r(0) & =1 .
\end{array}
$$

$f(\varkappa)$ is essentially the $(\varkappa Q)$-component of the piezoelectric ac field.

\section{Diagrammatic Analysis}

In this section we assume that the fields $f$ are probabilistic quantities which are subject to a Gaussian random process. We study the consequence of this by a systematic iterative treatment of Eq. (2).

To explain the method, the first iteration steps are described in detail. We associate with $r(v)$ a line which we picture as follows:

$$
r(v): \Longrightarrow=\text {. }
$$

The propagators $S(v)$ shall be represented by:

$$
S(v):
$$

and the fields $f(x)$ by:

$$
f(x):-\times-
$$

A vertex represented by a dot which joins three of these lines (but one and only one $f$-line) shall indicate summation over wave vectors of the outgoing lines conserving total wave vector. Iteration then is graphically represented by the following series:

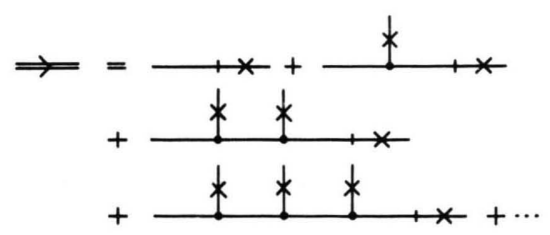

By performing the RPA, two $f$-lines join together:

$$
\left\langle f(x) f\left(x^{\prime}\right)\right\rangle=\delta_{x,-x^{\prime}} f(x) f(-x):-----
$$
and one gets

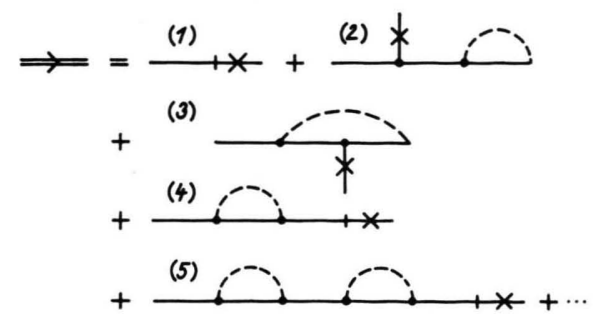


Clearly, only diagrams containing an even number of vertices will survive. Before we proceed to infinite diagram summation, a classification of diagrams is useful. We call a diagram reducible if it is divided into two by cutting one $S$-line or if it ends with an $S$-f-line combination, otherwise it is called irreducible. Diagrams (2), (4) and (5) are reducible. Furthermore, diagram (2) contains a closed loop. The $S$-line in front of this loop would be associated with the wave vector zero. But for $\varkappa=0$ our iterative equation is redundant. Such anomalous diagrams must therefore be omitted.

As there is no small coupling constant involved in the nonlinear term of Eq. (2), finite iterations are supposed to be inappropriate. A very simple infinite summation is obtained by

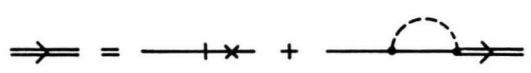

This is not a very deep result, since it follows by a single step iteration of the full Eq. (2) followed by the RPA.

Note, that diagrams of type (3) do not appear in this kind of iterative treatment. This is due to the fact, that the exact $r$-line is treated as a deterministic quantity.

A nontrivial extension of the theory is achieved if the $S$-lines are renormalized. We introduce the exact propagator $S^{\prime}$ defined by $r=S^{\prime} f$ :

$$
S^{\prime}(v):=
$$

It is noted that $S^{\prime}$ is connected to the nonlinear electronic response function $\varepsilon_{\mathrm{nl}}$ by the relation

$$
S^{\prime}(\varkappa)=\frac{1}{\varkappa} \frac{\varepsilon_{\mathrm{nl}}-1}{\varepsilon_{\mathrm{TF}}-1},
$$

where $\varepsilon_{\mathrm{TF}}=1+4 \pi e^{2} n_{0} /\left(\varepsilon_{0} k_{\mathrm{B}} T(\varkappa Q)^{2}\right)$ is the Thomas-Fermi dielectric constant of the crystal.

The approximation to $S^{\prime}$ corresponding to the above diagrams for $r$ is:

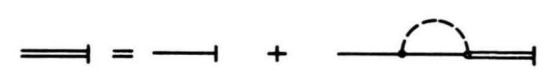

This still is a linear equation and will not lead very far. The next step is to insert diagrams into the irreducible "self energy" part covers $\leftrightarrows$. If we stop at this stage of approximation we end up with the following equation for $S^{\prime}$ :

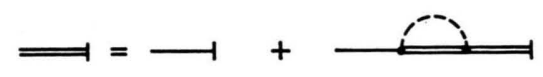

The analytic form of this diagrammatic equation is $S^{\prime}(v)=\frac{S(v)}{1-S(v) \sum_{x+\chi^{\prime}=v}^{\prime} S^{\prime}(\varkappa) f\left(\varkappa^{\prime}\right) f\left(-\varkappa^{\prime}\right)}$.

Equation (5) is nonlinear. It is supposed to be the simplest nontrivial integral equation for the propagator $S^{\prime}$ within the RPA. We note, that diagrams of type (3) are not accounted for by Eq. (5). Such diagrams are irreducible while Eq. (5) is the resummation result of reducible diagrams. We have pointed out, however, that the first kind of diagrams appears only if the exact $r$-lines are treated probabilistically. It is not clear whether this would be an improvement over the above treatment or not.

Finally it is noted that vertex renormalizations have been omitted completely. They would enhance the degree of nonlinearity and reduce the chance of an appropriate further treatment of the resulting nonlinear equation.

\section{An Application}

We base the following considerations on Eq. (5). It may be written in the form

$$
S^{\prime}(v)^{-1}=S(v)^{-1}-\sum_{x+x^{\prime}=v}^{\prime} S^{\prime}(\varkappa) f\left(\varkappa^{\prime}\right) f\left(-\varkappa^{\prime}\right) .
$$

Remembering the definition of $S$, it is seen, that

$$
-\sum_{x+x^{\prime}=\nu}^{\prime} S^{\prime}(\varkappa) f\left(\varkappa^{\prime}\right) f\left(-\varkappa^{\prime}\right)
$$

amounts to a renormalization of the quantity

$$
-i\left(1-v_{\mathrm{d}} / v_{\mathrm{s}}\right) \omega_{\mathrm{D}} / \omega(Q) .
$$

The renormalization contained in the $\mathrm{d} c$-term corresponding to $v=0$ is expected to come from the acoustoelectric current. Indeed, it is easy to show that

$$
-\sum_{\varkappa+x^{\prime}=0}^{\prime} S^{\prime}(\varkappa) f\left(\varkappa^{\prime}\right) f\left(-\varkappa^{\prime}\right)
$$

is identical to

$$
i \frac{\omega_{\mathrm{D}}}{\omega(Q)} \frac{j_{\mathrm{dc}}^{\mathrm{ae}}}{e n_{0} v_{\mathrm{s}}}
$$

where $j_{\mathrm{dc}}^{\text {ae }}$ is the acoustoelectric dc current density. This result was first found by YAMADA ${ }^{2}$ using the second order expression for $j_{\mathrm{dc}}^{\mathrm{ae}}$. It is seen, that this result is true to higher orders of perturbation theory, too. 
We therefore may account for this simply by choosing the correct value for $v_{\mathrm{d}}$. If one considers only a small volume element inside the crystal the correct form for $v_{\mathrm{d}}$ is clearly $v_{\mathrm{d}}=\mu E_{\mathrm{d}}$, where $\mu$ is the electron mobility and $E_{\mathrm{d}}$ the local dc electric field.
A more careful analysis of this question is necessary, however, if this "local" point of view is abandoned.

For an explicit treatment of Eq. (5) we write it as a continued fraction and go back to the quantity $r(v)$ :

$$
r(v)=S(v) f(v) /\left\{1-S(v) \sum_{x+x^{\prime}=v}^{\prime} S(x) f\left(x^{\prime}\right) f\left(-x^{\prime}\right) /\left[1-S(x) \sum_{x^{\prime \prime}+x^{\prime \prime \prime}=x}^{\sum^{\prime}=} \frac{S\left(\varkappa^{\prime \prime}\right) f\left(\varkappa^{\prime \prime \prime}\right) f\left(-x^{\prime \prime \prime}\right)}{1-S\left(\varkappa^{\prime \prime}\right) \sum^{\prime} \cdots}\right]\right\}
$$

This relation follows by infinite insertion of Eq. (5) into itself.

Equation (6) may be evaluated to yield a closed analytic expression under the following assumption: All $f$-modes which are sufficiently excited are concentrated in a small band around the mean wave vector $(\bar{v} Q)=\bar{k}$. The evaluation of an expression like

$$
S(v) \sum_{x+x^{\prime}=v} S(\varkappa) f\left(\varkappa^{\prime}\right) f\left(-\varkappa^{\prime}\right) /(1-g(\varkappa)),
$$

where $(\nu Q)$ is a wave vector in the small band around $(\bar{\nu} Q)$ then proceeds in the following way: In the $\varkappa^{\prime}$-sum, only the terms with $\varkappa^{\prime} \approx-\bar{v}$ are retained. Thus $x \approx 2 \bar{v}$ and one gets approximately

$$
S(v) \cdot S(2 \bar{v}) \sum_{\varkappa^{\prime}} f\left(\varkappa^{\prime}\right) f\left(-\varkappa^{\prime}\right) /(1-g(2 \bar{v})) .
$$

From the definition of $f\left(\varkappa^{\prime}\right)$ it follows

$\sum_{\varkappa^{\prime}} f\left(x^{\prime}\right) f\left(-x^{\prime}\right) \rightarrow-\bar{v}^{2} \sum_{x^{\prime} \approx \nu}\left|\tilde{\varphi}\left(\varkappa^{\prime} Q\right) / k_{\mathrm{B}} T\right|^{2}=-\bar{v}^{2} y^{2}$

where we have introduced the bunching parameter

$$
y=\sqrt{\sum_{x \approx \nu}\left|\tilde{\varphi}(\varkappa Q) / k_{\mathrm{B}} T\right|^{2}} .
$$

Furthermore,

$$
\bar{v}^{2} S(v) S(2 \bar{v}) \rightarrow \frac{1}{1-i \chi} \frac{1}{2-i \chi}
$$

where

$$
\chi=\frac{\omega_{\mathrm{D}}}{\omega(\bar{k})}\left(1-\frac{v_{\mathrm{d}}}{v_{\mathrm{s}}}\right)
$$

is the usual definition of the activity parameter.

The function $g(2 \bar{v})$ contains $S^{\prime}(2 \bar{v})$. But in our model this is not a well defined quantity from the physical point of view, since piezoelectric fields exist only in the wave vector band around $(\bar{v} Q)$. Thus we are free to impose a condition in which way the members of the set $\left\{S^{\prime}(n \bar{v}) ; n \geqq 2\right\}$ are connected to one another. We postulate that $S^{\prime}(n \bar{v})$ is determined via Eq. (5) by $S^{\prime}((n+1) \bar{v})$ alone. This amounts to retaining only terms with $x^{\prime} \approx-\bar{v}$ in sums of the form $\sum_{x+x^{\prime} \approx n} S(x) f\left(x^{\prime}\right) f\left(-x^{\prime}\right)$.

Rewriting all terms in the continued fraction in this way, one finds:

$$
\tilde{\varphi}(k)=n_{0} \frac{\tilde{\varphi}(k)}{k_{\mathrm{B}} T} \frac{1}{1-i \chi} \cdot 1 /\left[1+\frac{y^{2}}{(1-i \chi)(2-i \chi)\left(1+\frac{y^{2}}{(2-i \chi)(3-i \chi)\left(1+\frac{y^{2}}{\ldots}\right)}\right)}\right]
$$

We have reintroduced the physical quantities $\varrho$ and $\tilde{\varphi}$ associated with Eq. (1). From the theory of Bessel-functions ${ }^{8}$, it is known, that this continued fraction represents the function

$$
\frac{1-i \chi}{i y} \cdot \frac{J_{1-i \chi}(2 i y)}{J_{-i \chi}(2 i y)} .
$$

8 G. N. Watson, A Treatise on the Theory of Bessel Function, Cambridge University Press 1958.

9 W. Wonneberger, H. G. Reik, and A. Knoll, Phys. Lett. 30 A, 47 [1969].
Thus we find the explicit solution

$$
\tilde{\varrho}(k)=n_{0} \frac{\tilde{\varphi}(k)}{k_{\mathrm{B}} T} \frac{1}{i y} \frac{J_{1-i \chi}(2 i y)}{J_{-i x}(2 i y)} .
$$

This equation is identical in form to a former result on the electron bunching by a single coherent sound wave ${ }^{9, *}$. But the physical situation is quite

* A similar result on coherent electron bunching has also been found by GAY and HARTNAGEL ${ }^{10}$.

10 R. K. L. Gay and H. G. Hartnagel, Brit. J. Appl. Phys. 2, 1589 [1969]. 
different now. $\tilde{\varrho}(k)$ is the density fluctuation associated with the comparatively weak mode $\tilde{\varphi}(k)$ inside a frequency band containing a very large number of modes. All these $\tilde{\varphi}$-modes collectively influence each single $\tilde{\varrho}$-mode by their contribution to the bunching parameter $y$.

The nonlinear dielectric function following from Eq. (3) is again found to be given by

$$
\varepsilon_{\mathrm{nl}}=1+\left(\varepsilon_{\mathrm{TF}}-1\right) \frac{1}{i y} \frac{J_{1-i \chi}(2 i y)}{J_{-i \chi}(2 i y)} .
$$

The physical interpretation of Eq. (10) has changed in the same way as that of the $\tilde{\varrho}-\tilde{\varphi}$-relation (9). From $\varepsilon_{\mathrm{nl}}$ the acoustoelectric gain and the renormalization of the sound velocity follow in the same manner as derived in Ref. ${ }^{7}$ for a coherent sound wave.

\section{Discussion}

The general diagrammatic method of Section 2 was used to establish a nonlinear integral equation for the propagator $S^{\prime}$ which is intimately related to the nonlinear dielectric response function of a collision dominated solide state plasma.

Within the frame work of this equation we were able to extend an earlier result on nonlinear co-

11 W. Wonneberger and M. Schulz, Z. Naturforsch. 26 a, 1005 [1971].

12 W. Wonneberger, F. Siebert, and W. Wettring, Optics Comm. 3, 179 [1971].

13 M. Schulz and W. Wonneberger, Phys. Stat. Sol. (a) 3, 721 [1970].

14 M. Schulz, private communication. herent sound amplification to a simple situation of incoherent amplification of noise.

This extension has been presupposed in Ref. ${ }^{7}$. It is essential for the interpretation of the experimental results in Ref. ${ }^{11}$ and Ref. ${ }^{12}$. This correspondence was tested experimentally for stationary acoustoelectric domains in $\mathrm{GaAs}^{13}$ and found to be a reasonably good approximation. For travelling domains in $\mathrm{GaAs}$, it is found appropriate, too ${ }^{\mathbf{1 4}}$.

The full merit of the general approach presented in this paper is expected, however, in the complementary situation namely when the relevant modes are spread over a wide frequency range.

Finally it is pointed out that even subharmonic generation ${ }^{15,16}$ may be studied within this general scheme provided the RPA is not carried through with full consequence. One is then able to retain phase relations between modes which are expected to interact parametrically while the great mass of modes is still treated in the RPA. The phase relations may be taken into account by using the method of REIK et al. ${ }^{17}$.

\section{Acknowledgement}

The author is indebted to Prof. H. G. REIK and Dr. M. ScHulz for many helpful discussions.

15 J. Zucker, S. Zemon, and J. H. Wasko, Intern. Conf. on II-VI Semiconducting Compounds, 919 [1967], Benjamin, New York 1967.

16 M. Schulz and B. K. Ridley, Phys. Lett. 29 A, 17 [1969].

17 H. G. Reik, R. Schirmer, and H. HinkelmanN, Sol. State Comm. 7, 1309 [1969]. 